\title{
Evaluation of the Human Factor in the Scheduling of Smart Appliances in Smart Grids
}

\author{
Jânio Monteiro ${ }^{1,2}$, Pedro J.S. Cardoso ${ }^{1}$, Rita Serra ${ }^{1}$, and Licínia Fernandes ${ }^{1}$ \\ ${ }^{1}$ ISE, University of Algarve, Portugal \\ ${ }^{2}$ INOV, Lisbon, Portugal \\ jmmontei@ualg.pt
}

\begin{abstract}
Recently there has been an increase of interest in implementing a new set of home appliances, known as Smart Appliances that integrate Information Technologies, the Internet of Things and the ability of communicating with other devices. While Smart Appliances are characterized as an important milestone on the path to the Smart Grid, by being able to automatically schedule their loads according to a tariff or reflecting the power that is generated using renewable sources, there is not a clear understanding on the impact that the behavior of such devices will have in the comfort levels of users, when they shift their working periods to earlier, or later than, a preset time. Given these considerations, in this work we analyse the results of an assessment survey carried out to a group of Home Appliance users regarding their habits when dealing with these machines and the subjective impact in quality caused by either finishing its programs before or after the time limit set by the user. The results of this work are expected to be used as input for the evaluation of load scheduling algorithms running in energy management systems.
\end{abstract}

Keywords: Smart Grids, Home Grids, Human Factor, Comfort Level, Smart Appliances, Mean Opinion Score.

\section{Introduction}

Currently we are witnessing an increase in the energy produced by renewable sources, either motivated by the increase in the cost of oil exploitation or by an increment in environmental concerns, with its higher expression in the energy obtained from wind and photovoltaic sources. In this context, the traditional view of a distribution grid that uses centralized generators to provide power to consumers is being replaced by a "smart grid" solution where energy production is based in a Distributed Generation (DG) [1]. The power generated by these energy sources however varies according to environmental conditions which are not controllable. In this scenario the traditional role of consumers is being replaced by a more proactive one, not only in the sense that they should be able to produce energy locally, for self-consumption and/or feed it into the electrical grid, but also in the sense that they are expected to adjust their demand according to the power that is being produced. 
In this context, several scientific works [2][3][4] have analyzed the importance of an efficient management of home grids by consumers, or by an Energy Consumption Scheduling device (ECS). The role of an ECS is to optimally schedule loads in order to better harness the energy produced locally or shift them to work at the periods of time when the rate is lowest, while reflecting user preferences. This also requires that loads, like HVAC (heating, ventilation and air conditioning) and Home Appliances, should be able to communicate with the ECS device and shift their working periods, or adjust the power they consume, according to the power generated locally from renewable sources, or according to a supplier's tariff, which in turn may change dynamically.

By creating a new range of appliances that integrate Information Technologies, the Internet of Things (IoT) [5], with the ability of communicating and respond dynamically to the varying tariffs, a reduction on $\mathrm{CO}_{2}$ emissions to the atmosphere is possible [6], while ensuring at the same time higher returns on investments made in renewable energy sources. Due to this, as described in [7], Smart Appliances are characterized as an important milestone on the path to the Smart Grid.

In this scenario, one important feature associated with the ECN is to prevent electrical overloads that may happen when several appliances are scheduled to work in the same period of time in the search for a lower cost or tariff. In this case the ECN should decide which appliance is expected to work first and which ones should be scheduled to work later, reflecting a level of priority that should be commensurate with the user preferences. In fact, while overloads may prevent the user from using the ECN, the quality of the scheduling algorithm from a user perspective will also determine how it will be used.

While the shifting of the working periods of some of equipment do not have a clear correlation with the comfort levels of users, like for instance a swimming pool pump, the changing of the working periods of other appliances - like clothes washing machines, clothes dryer and dish washer - have a direct impact in people's routines and therefore affect the perception of the quality of the ECS algorithm. In fact, while the efficiency of a demand side management (DSM) algorithm that runs in an ECS is proportional to the level of flexibility that users impose to their appliances, we believe that that flexibility will depend on the correct assessment of user preferences made by the ECS.

Since an optimum or nearly optimum solution in terms of cost may imply changing the work period of the machine to several hours before the limit, or make it finish some minutes after the pre-set time limit, the Objective or Multi-objective Function of the scheduling algorithm should include an assessment of user preferences.

While the human factor in the scheduling of residential loads has been included in some algorithms [8][9], as far as we know there isn't any study that clearly defines the comfort level curves of the major appliances according to their time variation.

Given these considerations, in this work we analyze the results of an assessment survey carried out to a group of 44 users, concerning their habits and the subjective impact in quality that the clothes washers, clothes dryer and dish washer appliances have, when they finish their programs before and after a pre-set time limit. The results 
of this work are expected to be used in the evaluation of load scheduling algorithms running in ECS devices.

The rest of this paper is organized as follows. Section 2 describes the methodology used in the subjective assessment of user preferences. Section 3 presents the obtained results comprising the frequency and time-of-use of the appliances, distribution of type of tariffs, value given to an ECS device and assessment of the impact in quality caused by changing the moment when appliances finish their programs. Section 4 concludes the paper, pointing out future developments of this work.

\section{Methodology Used in the Subjective Assessment Survey}

In order to evaluate the user habits and its preferences when managing of home appliances, an online based survey was created covering the following issues: the assessor's frequency in using home appliances, time of use of such appliances, type of electrical tariff contracted with the electricity provider, the level of importance given to a device that controls home appliances and the subjective impact in quality caused by changing the moment when appliances finish their programs.

For the evaluation of the subjective impact caused by changing the moment when a clothes washer, clothes dryer or dish washer finishes its program a five-grade scale was used with each level mapping to a certain quality, as follows: 1- Bad Quality; 2Poor Quality; 3- Fair Quality; 4- Good Quality and 5- Excellent Quality. As users are expected to have different levels of tolerance to delay according to their daily routines, different conditions were evaluated comparing usage at week versus weekend days and at distinct times of the day.

The results of these tests were afterwards analyzed by obtaining the Mean Opinion Score (MOS) for each appliance and condition, using equation (1):

$$
\bar{u}_{m c t}=\frac{1}{N} \sum_{1}^{N} u_{i m c t}
$$

In equation (1), $\mathrm{Nm}$ represents the number of observers that use appliance $\mathrm{m}$, and uimct represents the score of observer $\mathrm{i}$, for appliance $\mathrm{m}$, in condition $\mathrm{c}$ and delta time t. We have considered the values of delta time $t$ to be negative (i.e., $t<0$ ) when the appliance finishes its program before the time limit set by the user, positive when it finishes after the limit set by the user, or zero when it finishes the program at the limit set by the user.

The MOS results obtained from equation (1) were afterwards used to obtain a regression function. The function used for regression was:

$$
f(t)=\alpha+e^{\beta+\chi t}
$$

The values of $\alpha, \beta$ and $\chi$ were obtained minimizing the distance between equation (2) and the set of umct points. For each appliance $m$ and condition $c$, two regression functions were considered, one for $\mathrm{t} \leq 0$ and another for $\mathrm{t} \geq 0$.

In the following section we present the results obtained from the assessment survey. 


\section{$3 \quad$ Results and Analysis}

The assessment panel which answered the survey was selected by invitation among retired persons, middle age individuals and college students, all of them in Portugal. The majority of questionnaires were answered online, resulting in a total of 44 answers.

\subsection{Frequency and Time-of-Use of the Appliances}

In the first question we have evaluated the frequency of usage of the clothes washers, clothes dryer and dish washer appliances by each individual. The results of these answers are shown in Table I.

Table 1. Frequency of usage of the clothes washers, clothes dryer and dish washer appliances

\begin{tabular}{|c|c|c|c|}
\hline \hline Assessor: & Clothes washer & Dish Washer & Clothes Dryer \\
\hline Does not use it & $0,0 \%$ & $\mathbf{2 5 , 0 \%}$ & $\mathbf{6 1 , 4 \%}$ \\
\hline Use it rarely & $2,3 \%$ & $2,3 \%$ & $\mathbf{2 5 , 0 \%}$ \\
\hline On average use it once per month & $4,5 \%$ & $2,3 \%$ & $6,8 \%$ \\
\hline On average use it once per week & $11,4 \%$ & $6,8 \%$ & $4,5 \%$ \\
\hline $\begin{array}{c}\text { On average use it two or three } \\
\text { times per week }\end{array}$ & $\mathbf{2 9 , 5 \%}$ & $20,5 \%$ & $0,0 \%$ \\
\hline $\begin{array}{c}\text { On average I use it more than } \\
\text { three times per week }\end{array}$ & $\mathbf{5 2 , 3 \%}$ & $\mathbf{4 3 , 2 \%}$ & $2,3 \%$ \\
\hline \hline
\end{tabular}

As it can be verified, among the three appliances covered in this study, the clothes washing machine is the most commonly used one, with nearly $82 \%$ of the subjects using it more than twice per week. Regarding the clothes dryer on contrary only 2,3\% of the subjects use it more than twice per week, while it is not used at all by $61,4 \%$ of the persons that answered the survey.

Table 2. Time-of-use frequency of the appliances in weekdays (DW) and weekend (WE)

\begin{tabular}{|c|c|c|c|c|c|c|}
\hline \hline & \multicolumn{2}{|c|}{ Clothes washer } & \multicolumn{2}{c|}{ Dish Washer } & \multicolumn{2}{c|}{ Clothes Dryer } \\
\hline Time of Day & WD & WE & WD & WE & WD & WE \\
\hline From 7am to 10 am & $11,5 \%$ & $6,1 \%$ & $0,0 \%$ & $2,3 \%$ & $0,0 \%$ & $4,8 \%$ \\
\hline From 10am to 12am & $11,5 \%$ & $\mathbf{3 7 , 9 \%}$ & $5,7 \%$ & $4,5 \%$ & $8,3 \%$ & $14,3 \%$ \\
\hline From 12am to 6 pm & $11,5 \%$ & $\mathbf{3 0 , 3 \%}$ & $5,7 \%$ & $\mathbf{2 7 , 3 \%}$ & $8,3 \%$ & $\mathbf{2 8 , 6 \%}$ \\
\hline From 6 pm to 12 pm & $\mathbf{4 6 , 2 \%}$ & $15,2 \%$ & $\mathbf{6 2 , 9 \%}$ & $\mathbf{5 0 , 0 \%}$ & $\mathbf{4 1 , 7 \%}$ & $\mathbf{4 2 , 9 \%}$ \\
\hline From 12pm to 10am & $\mathbf{1 9 , 2 \%}$ & $10,6 \%$ & $\mathbf{2 5 , 7 \%}$ & $15,9 \%$ & $\mathbf{4 1 , 7 \%}$ & $9,5 \%$ \\
\hline \hline
\end{tabular}


In the following question we asked for the time-of-use frequency of these appliances, differentiating weekdays from weekends. Table 2 presents these results.

As it can be verified, while during weekdays these appliances tend to be used between $6 \mathrm{pm}$ and $7 \mathrm{am}$ (of the following day), at the weekend they tend to be used earlier. This is more clearly verified in the case of the clothes washing machine, which at weekends is mostly used between 10 am and $6 \mathrm{pm}$, while the other two appliances are predominantly used between midday and midnight.

\subsection{Type of Tariffs Distribution and Recognized Importance of an ECS Device}

As the results in the previous section may reflect the type of tariff that users have, we asked assessors to specify it. These results are presented in Figure 1. As verified, half of the assessors had a time of use type of tariff, either comprising two or three time periods.

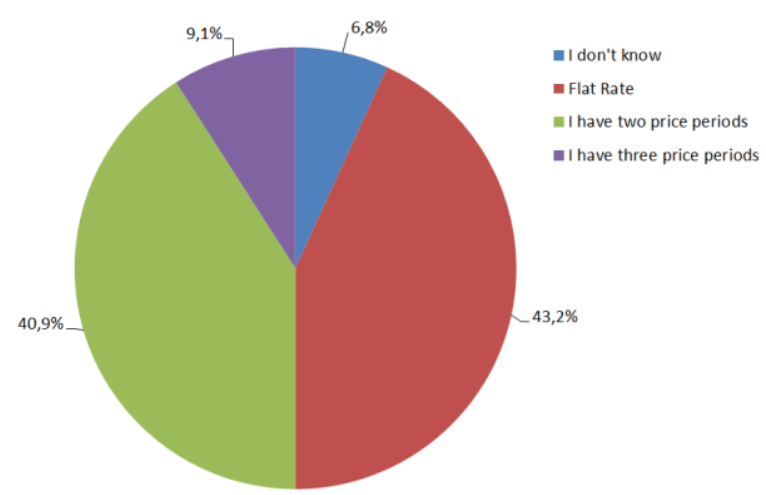

Fig. 1. Distribution of the electrical tariffs contracts among the assessment panel

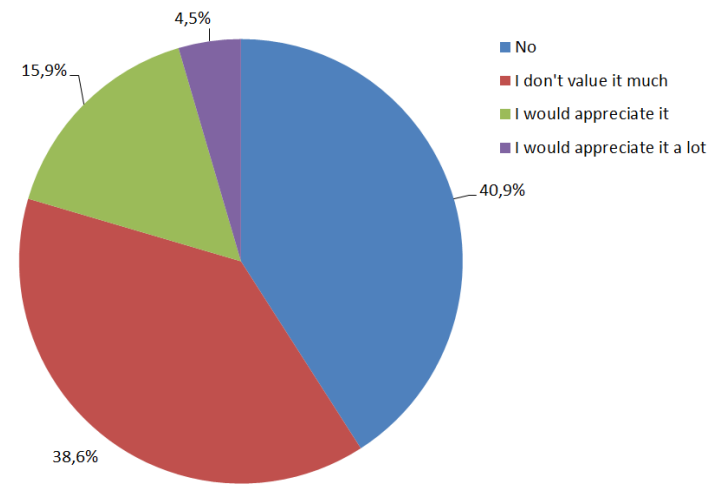

Fig. 2. Importance given to a device capable of managing appliances automatically 
When correlating the type of tariff with the time of use of the appliances, we verified that all of the assessors that use their appliances between midnight and 7 am had a time of use contract, driving them to change their routines.

When we asked users to state the level of importance that they recognize in having a device capable of scheduling their appliances automatically, around $45 \%$ of assessors didn't consider it relevant, as shown in Figure 2.However, among those users that have a time of use type of tariff (corresponding to nearly $50 \%$ of the survey universe), $95 \%$ of them considered it relevant. Among them $45 \%$ stated that they would appreciate it and $50 \%$ stated that they would appreciate it a lot.

\subsection{Assessment of the Impact in Quality Caused by Changing the Moment When Appliances Finish Their Programs}

In this section we evaluate the impact in quality (or comfort) that a scheduling device would have when changing the work period of appliances to end before or after the limit set by the user.

Before being asked to answer the following part of the survey, users were first instructed to consider a scenario in which they had a device in their homes capable of managing their appliances, so they would save money in electrical bills.

In the first group of questions we started by evaluating the impact in quality that a delay of either $7,15,30$ or 60 minutes would cause in a user, considering that the appliance was set to finish at a certain hour. As those impacts may depend on the type of appliance and on the time frame where the machine is expected to work, we asked assessors to consider different situations comprising: weekend days and either 7 am, 7 pm or $11 \mathrm{pm}$ in weekdays. These results are shown in Figures 3, 4 and 5, which represent the MOS results and the associated 95\% confidence intervals respectively for the clothes washers, clothes dryer and dish washer appliances.

In terms of the clothes washing machine, the results show that users tend to be less tolerant to delays at 7 am and $11 \mathrm{pm}$, when compared to $7 \mathrm{pm}$ and weekend days. Regarding the clothes dryer appliance the tolerance to delays tends to increase between $7 \mathrm{am}, 7 \mathrm{pm}$ and $11 \mathrm{pm}$ in weekdays and from those days to the weekend.

When comparing the three appliances, it was verified that users tend to be more tolerant with the delays caused by the dish washer, regardless of the moment when it is supposed to finish its program. The obtained difference between MOS of the dish washer at weekend days and the different hours of weekdays was small.

We have then evaluated the impact in quality of an appliance finishing sooner than the time limit set by the user. To measure it, we asked evaluators to grade the quality level of a machine ending either 8 hours, 4 hours, 2 hours, 1 hour or 30 minutes before the limit set by the user, or at the exact hour. Figure 6 presents the MOS and the associated $95 \%$ confidence intervals of these results for the three appliances analyzed in this study.

As it can be verified, users tend to be less satisfied when the clothes washing machine finishes much sooner than the limit, with a MOS of 2.26 for a 8 hours advance, translating to a subjective quality close to a 'Poor Quality' level. One of the reasons causing this dissatisfaction could be the fact that people don't like to leave clothes wet inside the washing machine for long periods of time. 


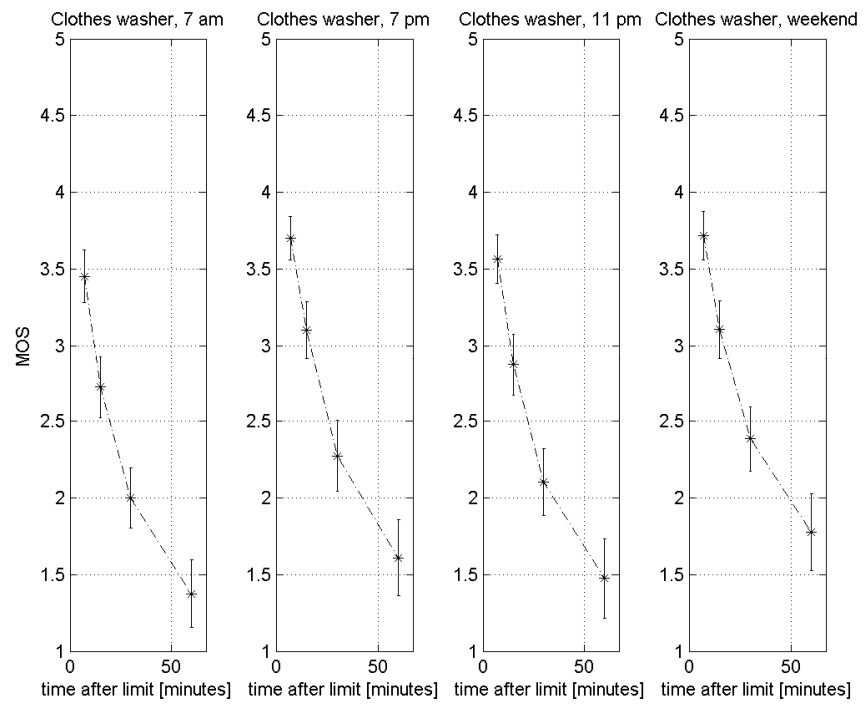

Fig. 3. MOS and associated $95 \%$ confidence intervals for the assessment of a clothes washing appliance finishing 7, 15, 30 and 60 minutes later than expected, at different hours and days of the week

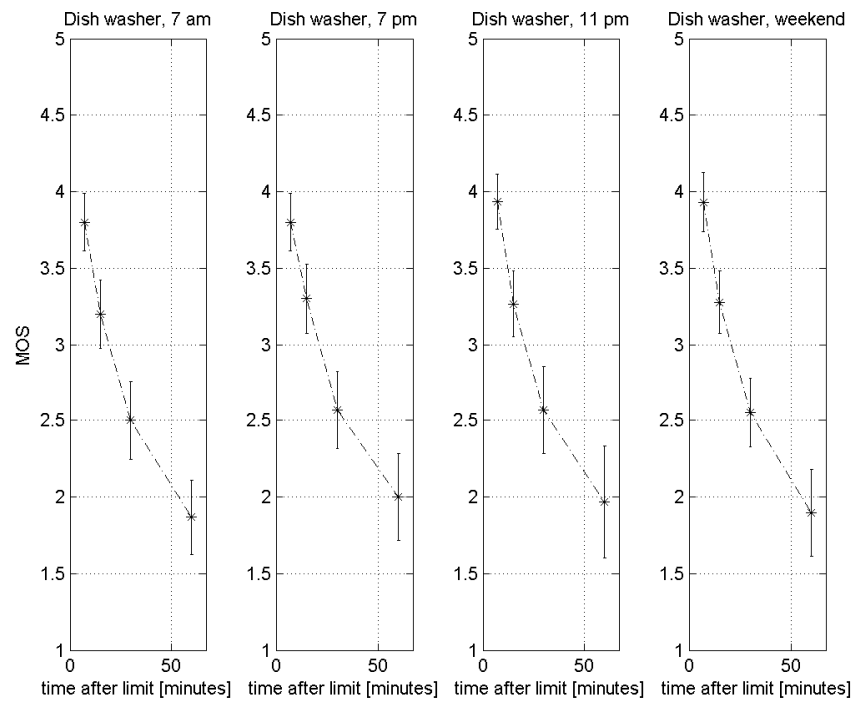

Fig. 4. MOS and associated $95 \%$ confidence intervals for the assessment of a dish washer appliance finishing 7, 15, 30 and 60 minutes later than expected, at different hours and days of the week 


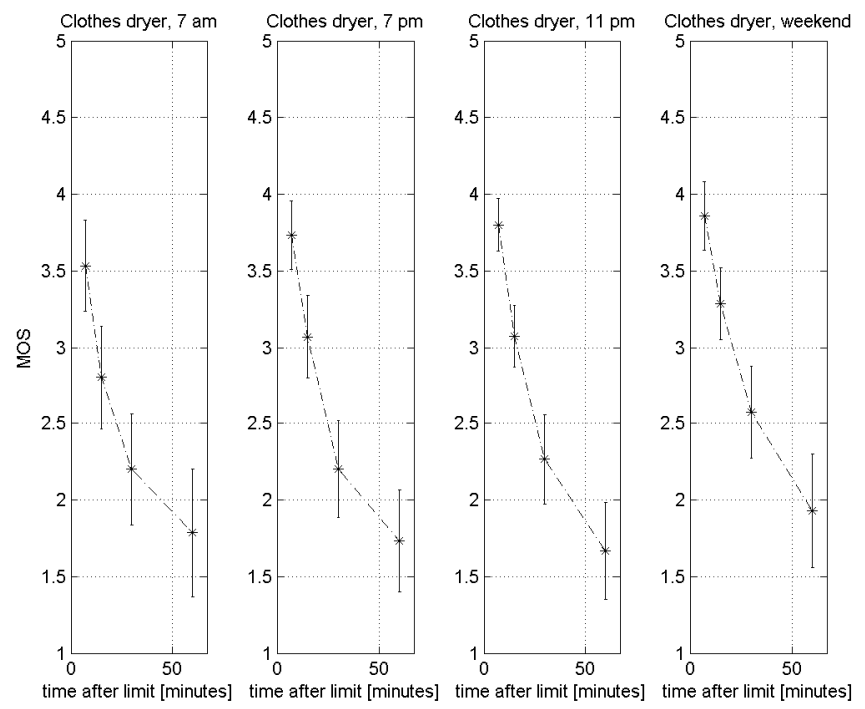

Fig. 5. MOS and associated $95 \%$ confidence intervals for the assessment of a clothes dryer appliance finishing 7, 15, 30 and 60 minutes later than expected, at different hours and days of the week

In terms of the dish washer machine, users tend to be more tolerant when it finishes much sooner than the expected time limit with a MOS of 2.75 for an 8 hours advance, slightly below the 'fair quality' level.

Regarding the clothes dryer appliance, while users do not penalize it as much as the clothes washing when it finishes significantly before the set limit, they tend to be more satisfied when it finishes exactly or little before the time limit.

We have also verified that while the majority of users consider that the clothes and dish washer appliances should finish their programs close to the end of the time limit, some users prefer them to finish their programs one or two hours before the limit, instead of ending up exactly at the threshold. This prevents the MOS results from reaching the Excellent Quality level (i.e., a MOS equal to 5.0), when the appliance finishes exactly at the predefined time limit.

Given these results, we have computed a regression function of the MOS values for each appliance using equation (2), considering an hour based time scale for all curves. These results are presented in Table 3.

Regarding the results obtained for the evaluation of late end of the programs, since we had different situations comprising weekend days and several hours of weekdays, we have computed a MOS that included all these answers. Using these new MOS points we have afterwards performed a new curve fitting. Figure 7 presents these MOS points, together with the curve fitting curves using equation (2) for the three types of appliances. Table 4 presents the regression results of these MOS points. 

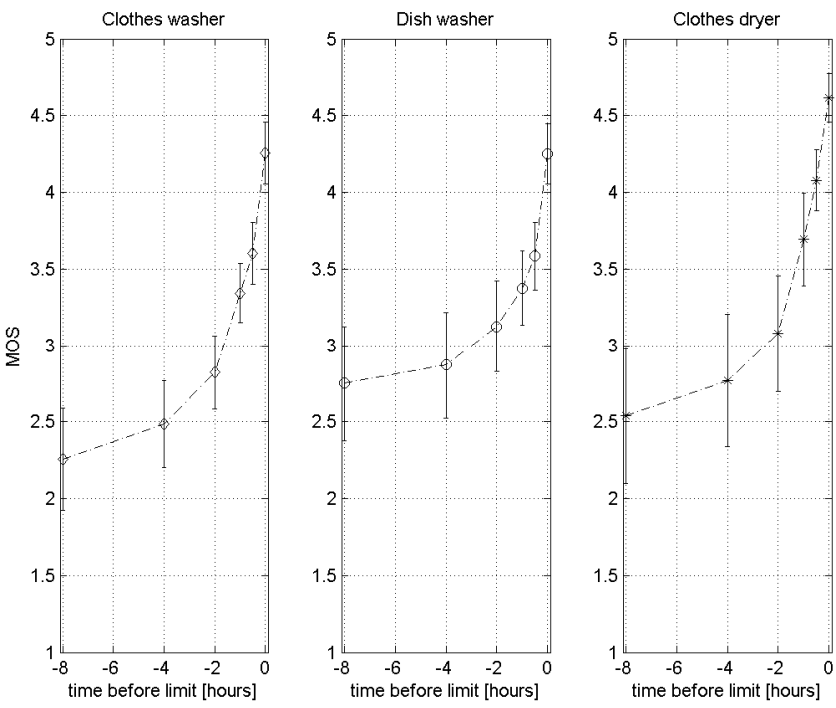

Fig. 6. MOS and associated $95 \%$ confidence intervals for the assessment of a clothes dryer, dish washer and clothes dryer appliances finishing sooner than the time limits set by the user

Table 3. Statistical parameters resulting from the regression of MOS points when the appliances finish their programs later than the set limit, in different time frames

\begin{tabular}{|c|c|c|c|c|}
\hline \multirow[b]{2}{*}{ Fitting Results } & \multicolumn{3}{|c|}{ Weekdays } & \multirow{2}{*}{ Weekend } \\
\hline & $7 \mathrm{am}$ & $7 \mathrm{pm}$ & Midnight & \\
\hline \multicolumn{5}{|c|}{ Clothes Washer } \\
\hline$\alpha$ & 1.1655 & 1.1389 & 1.1828 & 1.4077 \\
\hline$\beta$ & 1.1296 & 1.1480 & 1.1293 & 1.0575 \\
\hline$\chi$ & -2.6680 & -1.9437 & -2.3894 & -2.0964 \\
\hline Standard Deviation & 0.0278 & 0.0616 & 0.0356 & 0.05136 \\
\hline Correlation Coefficients & 0.99985 & 0.99916 & 0.99974 & 0.99934 \\
\hline \multicolumn{5}{|c|}{ Dish Washer } \\
\hline$\alpha$ & 1.4082 & 1.5535 & 1.4546 & 1.2878 \\
\hline$\beta$ & 1.0585 & 1.0051 & 1.0550 & 1.1109 \\
\hline$\chi$ & -1.8726 & -1.8610 & -1.7796 & -1.6507 \\
\hline Standard Deviation & 0.0677 & 0.0674 & 0.1286 & 0.1230 \\
\hline Correlation Coefficients & 0.99876 & 0.99863 & 0.99535 & 0.99596 \\
\hline \multicolumn{5}{|c|}{ Clothes Dryer } \\
\hline$\alpha$ & 1.7558 & 1.5505 & 1.4366 & 1.6690 \\
\hline$\beta$ & 1.0465 & 1.1234 & 1.1602 & 1.0756 \\
\hline$\chi$ & -3.9491 & -2.9447 & -2.6600 & -2.3944 \\
\hline Standard Deviation & 0.0435 & 0.0527 & 0.2226 & 0.0268 \\
\hline Correlation Coefficients & 0.99962 & 0.99948 & 0.99991 & 0.99984 \\
\hline
\end{tabular}




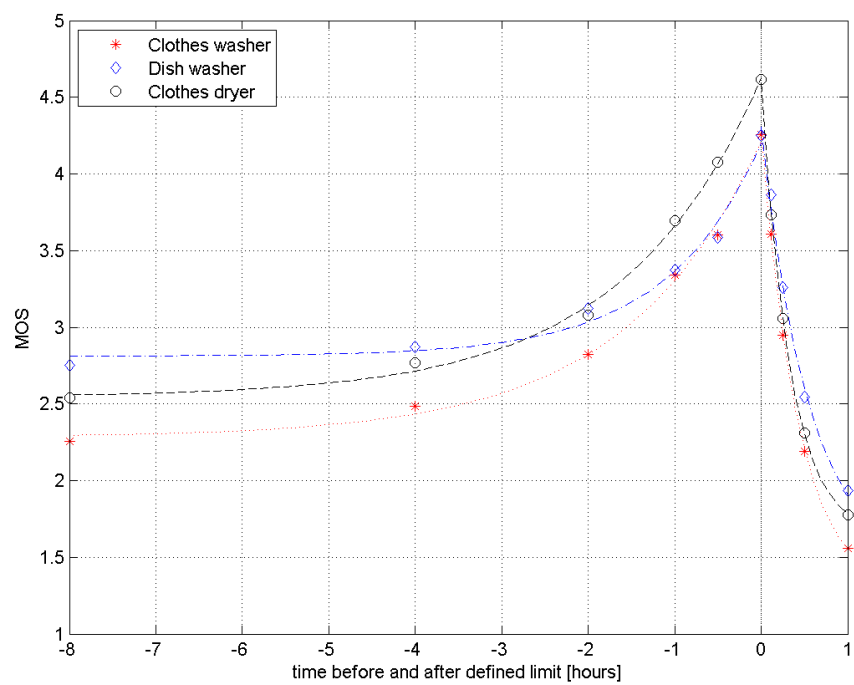

Fig. 7. MOS points and associated regression functions of the Clothes Washing, Dish Washer and Clothes Dryer appliances

The combined analysis of Figure 7 and Table 2, shows that these curves represent a good aproximation to the MOS points, with correlation coeficients higher than 0.99 .

Table 4. Statistical parameters resulting from the regression of the MOS when the appliances finish Before the set Limit (BL) and After the set Limit (AL)

\begin{tabular}{|c|r|r|r|r|r|r|}
\hline \hline & \multicolumn{2}{|c|}{ Clothes washer } & \multicolumn{2}{c|}{ Dish Washer } & \multicolumn{2}{c|}{ Clothes Dryer } \\
\hline Fitting Results & \multicolumn{1}{c|}{ BL } & \multicolumn{1}{c|}{ AL } & \multicolumn{1}{c|}{ BL } & \multicolumn{1}{c|}{ AL } & \multicolumn{1}{c|}{ BL } & \multicolumn{1}{c|}{ AL } \\
\hline$\alpha$ & 2.28360 & 1.2361 & 2.81172 & 1.4296 & 2.54453 & 1.6253 \\
\hline$\beta$ & 0.65824 & 1.1122 & 0.32819 & 1.0568 & 0.73273 & 1.0940 \\
\hline$\chi$ & 0.63582 & -2.2738 & 0.91504 & -1.7900 & 0.62390 & -2.9560 \\
\hline Standard Deviation & 0.07081 & 0.03578 & 0.09419 & 0.0938 & 0.05393 & 0.0084 \\
\hline Correlation Coefficients & 0.99732 & 0.99972 & 0.99111 & 0.99754 & 0.99865 & 0.9999 \\
\hline \hline
\end{tabular}

Table 5. Priority levels of the three appliances to be considered in scheduling algorithms

\begin{tabular}{|c|c|c|c|}
\hline \hline \multirow{2}{*}{ Priority } & \multicolumn{3}{|c|}{ Condition - Selecting Appliance to finish: } \\
\cline { 2 - 2 } & Much sooner & Near the pre-set time & Later than time limit \\
\hline High & Dish Washer & Clothes Dryer & Dish Washer \\
\hline Medium & Clothes Dryer & \multirow{2}{*}{$\begin{array}{c}\text { Dish Washer and } \\
\text { Clothes Washer }\end{array}$} & Clothes Dryer \\
\hline Low & Clothes Washer & Clothes Washer \\
\hline
\end{tabular}


Given these results, we have categorized the importance given to the appliances in three levels of priority (High, Medium and Low). These prioritization results are shown in Table 5 and may be considered by scheduling algorithms.

\section{Conclusions}

The main contribution of this paper include the results of the frequency, time-of-use distribution and the regression functions that measure the impact in quality caused by changing the moment when appliances finish their programs.

The high percentage of users with a time-of-use type of tariff that consider it relevant to have an ECS device capable of scheduling appliances automatically is a good indicator of the importance of such devices in the near future.

Regarding the tolerance to delays of the three appliances in this study, it was verified that users tend to be more tolerant with the delays of the dish washer, regardless of the moment when it is supposed to finish its program.

On the other hand, users tend to be less satisfied when the clothes washing machine finishes much sooner than the limit, with a MOS that is close to the Poor Quality level. Regarding the clothes dryer appliance, while users do not penalize quality as much as the clothes washer when it finishes significantly before the set limit, they tend to be more satisfied when it finishes exactly or little before the time limit.

Finally, the regression results of the MOS points using equation (2), show that the obtained functions and parameters have achieved a good approximation to the MOS points and thus can be considered a good representation of the human assessment of quality, in the scheduling of these appliances.

Acknowledgments. This work was supported by the Portuguese QREN R\&TD Project Number 30260, "Managing The Intelligence" (MTI).

\section{References}

1. Shen, S.: Empowering the Smart Grid with Wireless Technologies, Editor's Note. IEEE Network Magazine (May/June 2012)

2. Erol-Kantarci, M., Mouftah, H.T.: Wireless Sensor Networks for Cost-Efficient Residential Energy Management in the Smart Grid. IEEE Transactions on Smart Grid 2(2) (June 2011)

3. Infield, D.G., Short, J., Horne, C., Freris, L.L.: Potential for domestic dynamic demand-side management in the UK. Presented at the IEEE Power Eng. Soc. Gen. Meet., Tampa, FL (June 2007)

4. Stamminger, R.: Synergy potential of smart appliances Univ. Bonn, Bonn, Germany (March 2009), http: / / www . smart-a . org, Deliverable 2.3 of work package 2 from the SmartA project

5. What the Internet-of-things Will Mean for the Smart-grid. IEEE Smart Grid (June 2011), http: //smartgrid.ieee.org/june-2011/

95-what-the-internet-of-things-will-mean-for-the-smart-grid 
6. The Impact of Smart Grid Residential Energy Management Schemes on the Carbon Footprint of the Household Electricity Consumption. In: Erol-Kantarci, M., Mouftah, H.T. (eds.) 2010 IEEE Electrical Power \& Energy Conference (2010)

7. Arrival of Smart Appliances is a milestone on the path to the Smart Grid. IEEE Smart Grid (October 2011),

http://smartgrid.ieee.org/newsletter/october-2011/

415-arrival-of-smart-appliances-is-a-milestone-on-the-pathto-the-smart-grid

8. Esser, A., Kamper, A., Franke, M., Most, D., Rentz, O.: Scheduling of electrical household appliances with price signals. In: Operations Research Proceedings, pp. 253-258. Springer (2006)

9. Bozchalui, M., Hashmi, S., Hassen, H., Canizares, C., Bhattacharya, K.: Optimal Operation of Residential Energy Hubs in Smart Grids. To appear in IEEE Transactions on Smart Grid (July 2012) (accepted ) 